\title{
Effect of COVID-19 pandemic decisions on tertiary-level surgical services in Pietermaritzburg, KwaZulu-Natal Province, South Africa
}

\author{
D J Laäs, MB ChB, MMedSc; Z Farina, MB ChB, FCA; D G Bishop, MB ChB, FCA, PhD \\ Discipline of Anaesthesiology and Critical Care, College of Health Sciences, University of KwaZulu-Natal, Durban, South Africa
}

Corresponding author: D J Lä̈s (daniel.j.laas@gmail.com)

Background. The COVID-19 pandemic has led to the implementation of restrictive policies on theatre procedures, with profound impacts on service delivery and theatre output.

Objectives. To quantify these effects at a tertiary hospital in KwaZulu-Natal Province, South Africa.

Methods. A retrospective review of morbidity and mortality data was conducted. The effects on emergency and elective caseload, intensive care unit (ICU) admissions from theatre, theatre cancellations and regional techniques were noted.

Results. Theatre caseload decreased by 30\% from January to April 2020 ( $p=0.02)$, ICU admissions remained constant, and theatre cancellations were proportionally reduced, as were the absolute number of regional techniques.

Conclusions. The resulting theatre case deficit was 1260 cases. It will take 315 days to clear this deficit if four additional surgeries are performed per day.

S Afr Med J 2021;111(2):120-123. https://doi.org/10.7196/SAMJ.2021.v111i2.15332

The COVID-19 pandemic has caused a paradigm shift in how we manage and risk-stratify patients presenting for both elective and emergency surgery. ${ }^{[1]}$

Reports of a novel coronavirus causing severe acute respiratory syndrome first emerged from the city of Wuhan in the Hubei Province of China. ${ }^{[2]}$ It was officially reported by the World Health Organization China office on 31 December 2019. The first confirmed South African (SA) case of COVID-19 was identified in March 2020 in Hilton, KwaZulu-Natal (KZN) Province. Subsequently there has been a national drive to try to minimise the spread of the virus and reduce its impact on SA's healthcare system. This culminated in the declaration of a National State of Disaster and implementation of a forced public lockdown on 27 March. ${ }^{[3]}$

Grey's Hospital is a tertiary hospital in Pietermaritzburg, KZN. The hospital drains an estimated population of 3.5 million and provides tertiary surgical and critical care services to the western half of the province. ${ }^{[4]}$ The theatre complex has 10 operating theatres, including two dedicated 24-hour emergency theatres. On 25 March 2020, a directive was issued by hospital management (in accordance with national guidelines) to suspend all elective surgery at the hospital from 30 March.

As part of the national guidelines on management of the pandemic, theatre complexes have had to target: $(i)$ protection of patients and staff from exposure to the disease; (ii) facilitating redeployment of staff to manage an expected surge of COVID-19 patients; (iii) maximising hospital beds available for the predicted COVID-19 patient surge; and (iv) facilitating the redistribution of resources to contain the outbreak.

\section{Objectives}

The early effects of the policies implemented to prepare for the virus have not been described in a resource-limited theatre complex. The objective of this study was to evaluate and quantify the effect of
COVID-19 prevention and preparation policies on a tertiary hospital theatre complex in KZN by assessing changes in the number and type of theatre cases. Secondary outcomes included comparing the following before and after the implementation of restrictive theatre policies: ( $i$ ) theatre patient cancellations; (ii) regional techniques; and (iii) intensive care unit (ICU) admissions from theatre.

\section{Methods}

This was a retrospective, comparative, observational descriptive study conducted over 4 months (1 January - 30 April 2020). Weekly Department of Anaesthesia morbidity and mortality (M\&M) meeting data were collated and analysed. The M\&M data were collected from daily slate reports, including elective and emergency theatre slate reports. These reports list the following: ( $i$ ) details of patients booked for the slate; (ii) details of patients cancelled and the reason for cancellation; (iii) ICU admission from theatre, subdivided into planned and unexpected admissions, as well by destination ICU (neonatal, paediatric and adult); and (iv) regional or neuraxial procedures performed.

Data validity was improved by the following checks: (i) ICU admissions were verified against the theatre recovery room discharge book; (ii) regional techniques were compared with theatre nursing records; and (iii) all theatre cases at Grey's Hospital during the period of data collection were captured by retrospectively analysing theatre registers.

Relevant details from the theatre registers were entered into an Excel spreadsheet, version 16.43 (Microsoft, USA).

All surgical procedures performed in the hospital theatre complex were included in the study (obstetrics, gynaecology, general surgery, orthopaedics, ear, nose and throat surgery, maxillofacial surgery, paediatric surgery, trauma, burns, urology, ophthalmology, and emergencies from these disciplines). Procedures done in hospital wards, minor theatre or any location out of theatre were not included. 


\section{Statistical analysis}

Data were captured into an Excel spreadsheet for statistical analysis and imported into Stata release 15.1 (StataCorp, USA).

Counts of emergency and elective cases, adult, paediatric and neonatal ICU admissions, regional techniques and the number and ratio of theatre cancellations pre and post COVID-19 intervention policies were analysed. Percentages, together with the $95 \%$ confidence intervals (CIs), standard deviations (SDs), mean and median values, were obtained. The distribution of the data was assessed graphically, and measures of skewness and kurtosis were used to decide whether the data were parametric or nonparametric.

Analysis of the classification of procedures was done using descriptive statistics, and uniformly weighted moving averages of one lagged term, one forward term and the current term. A two-sample Wilcoxon rank-sum (Mann-Whitney) test was used to compare medians between the two periods. A $p$-value $<0.05$ was considered to be statistically significant. The null hypothesis was that no change occurred in theatre output after restrictive theatre policies were implemented. The primary outcome was that there was a significant reduction in cases after the implementation of restrictive policies.

\section{Ethical considerations}

Permission for this study was obtained from the University of KwaZulu-Natal's Biomedical Research Ethics Committee (ref. no. BREC/00001702/2020), the provincial Department of Health's ethical review board (ref. no. KZ_202008_052) and the CEO of Grey's Hospital.

\section{Results}

Over the 4 months of data collection, 2517 procedures were captured in M\&M data. For M\&M data, 1 234/2 517 procedures (49\%) were classified as emergency and 1 283/2 517 (51\%) as elective.

M\&M theatre caseload was divided into calendar weeks, except for the first and last week owing to the chosen sampling strategy. The mean (SD) number of cases done per week was 139.8 (34.40) (95\% CI 122.72 . 159.95) with the minimum being 60 and the maximum 182. These data are illustrated in Fig. 1, using rolling averages.

The total adult ICU admissions from theatre per week during the same period are illustrated in Fig. 2. Theatre cancellations and regional techniques are shown in Fig. 3. Table 1 compares theatre cases for January and April.

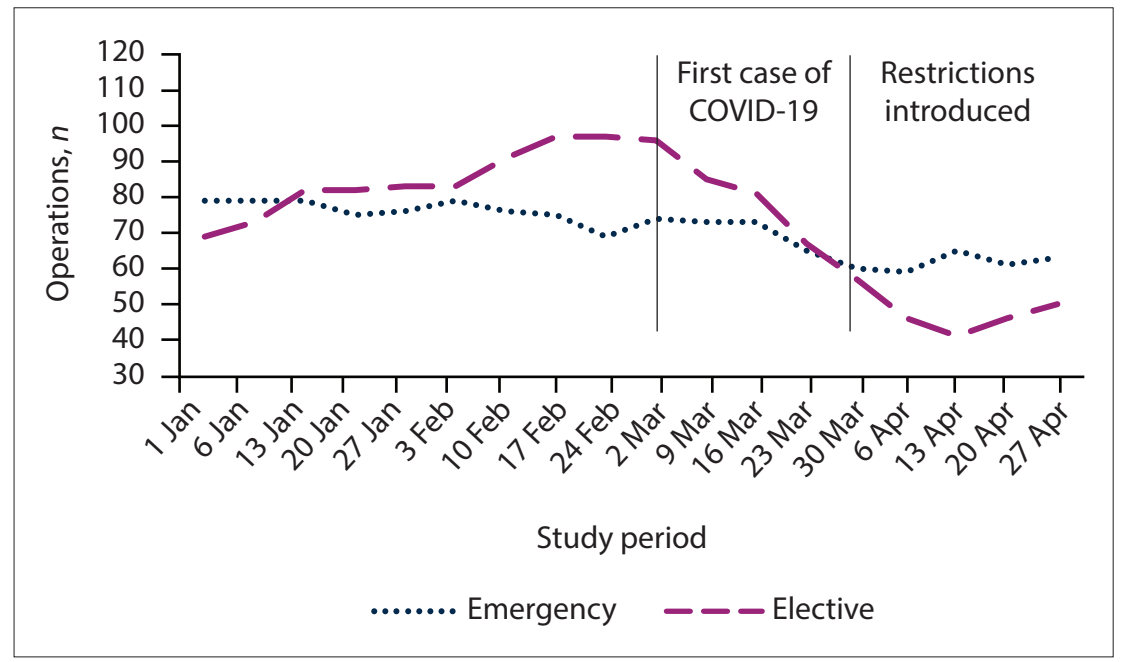

Fig. 1. Operations performed over the study period.

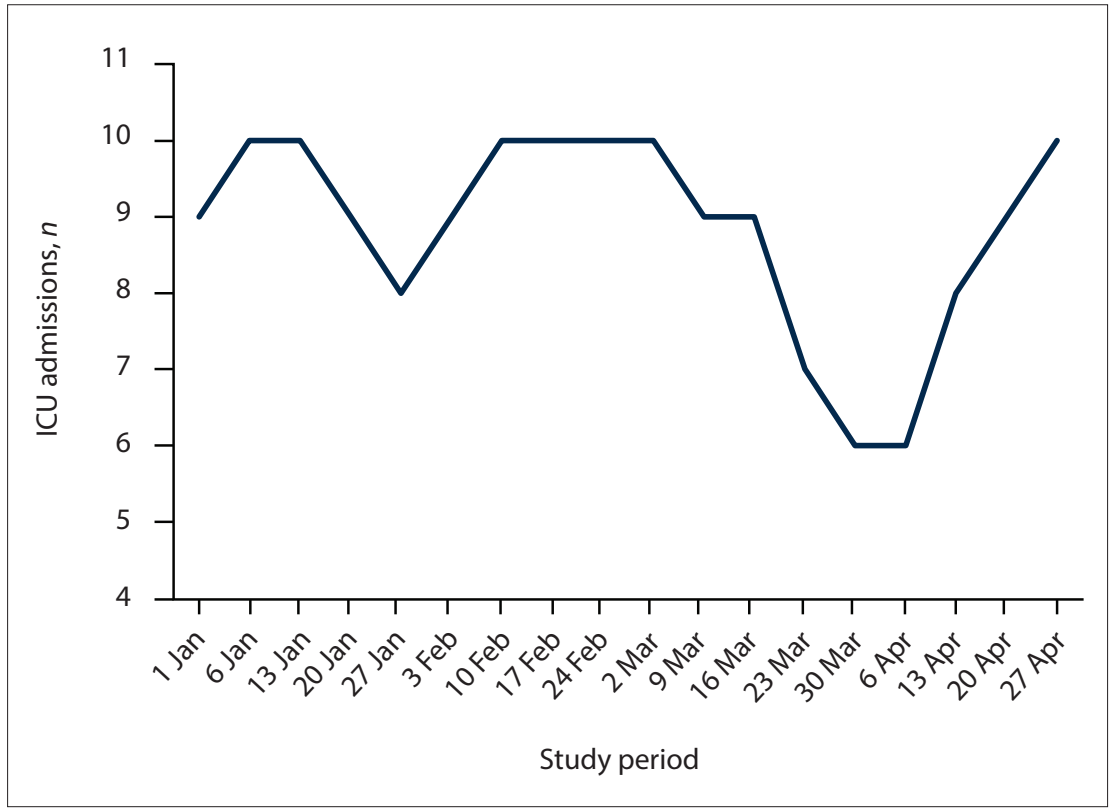

Fig. 2. Adult ICU admissions from theatre over the study period. (ICU = intensive care unit.)

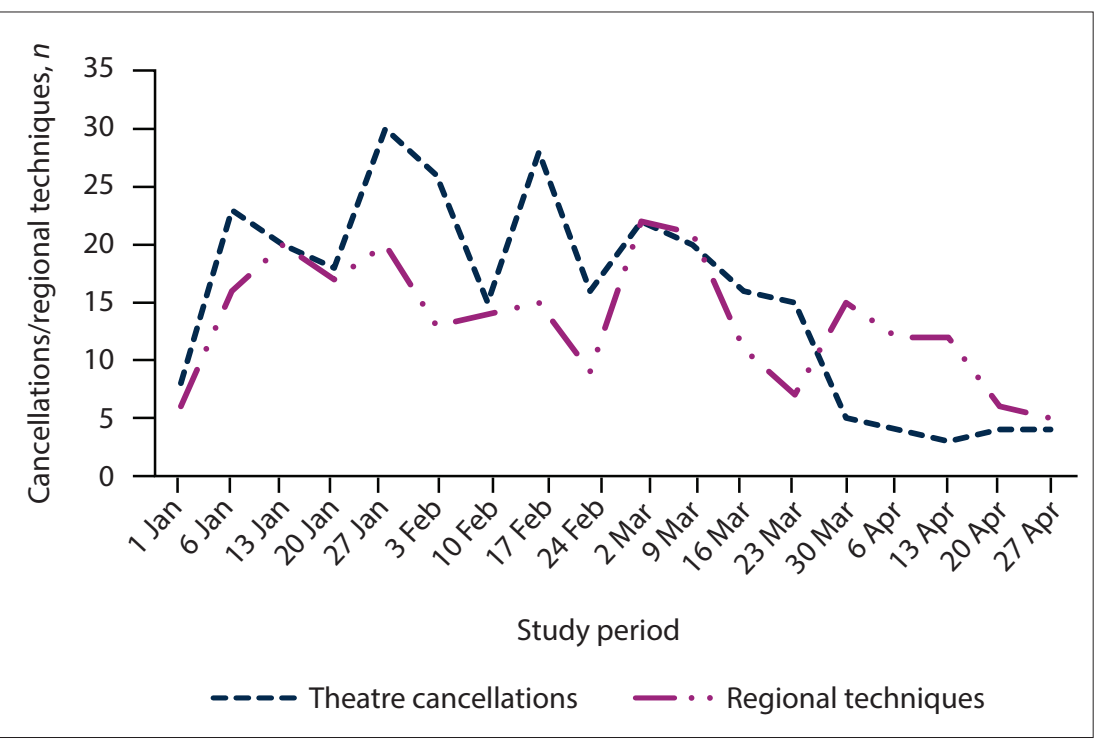

Fig. 3. Theatre cancellations and regional technique trends over the study period. 
Table 1. Effect of pandemic decisions, January v. April 2020

\begin{tabular}{|c|c|c|c|}
\hline & January (4 weeks) $(n)$, median (IQR) & April (4 weeks) (n), median (IQR) & $p$-value \\
\hline Emergency procedures & $78.8(76.9-79.2)$ & $61.8(60.2-63.9)$ & 0.02 \\
\hline Elective procedures & $77.5(71.0-82.2)$ & $46.0(43.5-48.2)$ & 0.02 \\
\hline Total procedures & $154.8(149.8-159.5)$ & $107(105.8-109.9)$ & 0.02 \\
\hline
\end{tabular}

\section{Discussion}

There was a statistically significant $30 \%$ decrease in all operations during the period analysed. During the pre-lockdown phase, the mean number of cases done at our institution was 700 per month. A 30\% reduction translates into 210 cases not performed during each month of restrictive theatre policies imposed. Extrapolating from the study period to include the whole period up to the commencement of level 1 lockdown restrictions (1 October 2020 - 6 months in total) reveals a 'total case deficit' of 1260 operations. To put this in pragmatic terms, should four out of the ten usual theatres do one additional patient per day, it would take 315 theatre days to clear this backlog (excluding Saturdays, Sundays and public holidays, this is equivalent to $\sim 1.3$ years). Given that theatre services were already constrained prior to COVID-19 policies, this is an optimistic time frame.

The decline in elective surgery noted was due to the implementation of restrictive theatre policies. The reason for the decrease in emergency surgery is suspected to be two-fold: firstly the national lockdown with the banning of alcohol sales and social gatherings, and secondly fewer elective cases, which resulted in fewer postoperative complications requiring repeat emergency surgery. ${ }^{[5]}$

We anticipated that the rate of ICU admissions from theatre would decline to a similar extent. However, the rate remained constant throughout the data collection period. This unexpected finding may have been due to delays in presentation caused by altered healthseeking behaviour of individuals during the lockdown. ${ }^{[6]}$

The case cancellation rate decreased to $5 \%$ of all cases from the pre-lockdown rate of $\sim 12 \%$. The most common reason for cancelling theatre cases at our institution is the overbooking of slates. This observation gives some additional insight into the general pressure that state-run surgical services are under in SA. ${ }^{[7]}$

This study was not designed to detect the long-term impacts of these restrictions, but they can be broadly characterised into health and training impacts. One aspect of the training impact can be seen in the reduction of utilisation of regional anaesthesia. Regional anaesthetic techniques were reduced by a rate similar to elective theatre cases.

In further evaluating the data, we found the classification of procedures challenging. The traditional division of theatre cases into 'elective' and 'emergency' has been of use in planning standard operative services. We attempted to reclassify theatre cases into more practical categories that will aid in clinical decision-making. However, the definitions of 'elective' and 'emergency' are unclear. These definitions do not take into account the clinical effect of postponements measured in months and years, but rather reflect triage between after-hours and daytime work. During retrospective reclassification, it was noted that there was a poor correlation between reviewers, and no reliable conclusion could be drawn. Final analysis was confined to the traditional classification. ${ }^{[8]}$

A recently published article on medically necessary timesensitive procedures proposed an elaborate scoring system based on procedure factors, disease factors and patient factors. ${ }^{[9]}$ This triage score has yet to be validated in our setting. A modification of the tool developed by Smith and colleagues (National Department of Health Technical Working Group on COVID-19 and Surgery, chairperson Prof. M D Smith, personal communication) has been circulated by the Working Group. This tool is in the process of being peer-reviewed and piloted, but it appears promising. We believe that this type of approach could have been more effective than traditional classification systems in the management of theatre services.

The primary and secondary outcomes showed the negative effects on service delivery of COVID-19 restrictive theatre policies. What our research does not show is the benefit obtained from implementing restrictions. It should be noted that where the public health response to the pandemic was delayed and inefficient, mortality rates among patients and healthcare workers were significant. ${ }^{[10,11]}$

\section{Study limitations and future research}

This study was effectively a pilot study conducted over a limited period. It leads the way for a follow-up multicentre study conducted over a more extended period to evaluate and compare the effects of restrictive theatre policies, the pandemic itself, the anticipated second surge and the recovery period. This follow-up study should include validation of the proposed triage tools.

In addition to the above, our research was not designed to show the effects of lockdown policies on individual surgical disciplines and their patients. Analysing these effects on different specialties can assist policymakers and theatre managers to better organise theatre systems, once surgical services revert to normal. By assigning more time to the most disadvantaged disciplines, it may be possible to distribute theatre resources appropriately.

Future research should also be conducted on the effect of COVID19 theatre policies on registrar and intern training.

\section{Conclusions}

This study is relevant to all district, regional and tertiary hospital theatre managers. The reduction in service delivery seen reflects the changes that occurred because of the planning and preparation phase of the COVID-19 pandemic. The unpredictable progression of the pandemic makes it challenging to anticipate when theatre complexes will revert to normal function. The lack of utility of the traditional emergency/elective subdivision of theatre cases is noted. An urgent area for future research is the validation of proposed new scoring systems, making them relevant to both traditional emergency triage and the longer timeline required when managing a pandemic. A better approach is needed to avoid unnecessary creation of a backlog in delivered surgical care.

Declaration. The research for this study was done in partial fulfilment of the requirements for DJL's MMed (Anaes) degree at the University of KwaZulu-Natal.

Acknowledgements. Dr R Laäs, Dr N Kheswa, Dr F Khatib, Dr E Gwala, Dr R Rodseth, C Connolly.

Author contributions. DJL: substantial contribution to conceptualisation, design, analysis and interpretation of data; ZF: critical revision; DGB: critical revision.

Funding. None.

Conflicts of interest. None. 
1. World Health Organization. Naming the coronavirus disease (COVID-19) and the virus that causes it. 2020. https://www.who.int/emergencies/diseases/novel-coronavirus-2019/technical-guidance/namingthe-coronavirus-disease-(covid-2019)-and-the-virus-that-causes-it (accessed 18 June 2020).

2. National Institute of Communicable Diseases. Coronavirus disease 2019 (COVID-19) quick reference for health workers. 25 May 2020. https://www.nicd.ac.za/wp-content/uploads/2020/05/COVID-19-
for for health workers. 25 May 2020. https./Www.incd.ac.za/wp

3. South Africa. Disaster Management Act No. 57 of 2002. https://sacoronavirus.co.za/2020/03/26/ South Africa. Disaster Management Act No. 57 of 2002 .
national-lockdown-regulations/ (accessed 18 June 2020).

national-lockdown-regulations/ (accessed 18 June 2020 ).
4. KwaZulu-Natal Department of Health. Grey's Hospital - a tertiary health care institution. http://www. kznhealth.gov.za/Greys/tertiary_health_institution.htm (accessed 18 June 2020).

5. Reuter H, Jenkins L, de Jong M, Reid S, Vonk M. Prohibiting alcohol sales during the coronaviru disease 2019 pandemic has positive effects on health services in South Africa. Afr J Prim Health Care Fam Med 2020;12(1). https://doi.org/10.4102/phcfm.v12i1.2528

6. Wong J, Cheung K. Impact of COVID-19 on orthopaedic and trauma service. J Bone Joint Surs 2020;102(14):e80. https://doi.org/10.2106/JBJS.20.00775

7. Nepogodiev D, Bhangu A. Elective surgery cancellations due to the COVID-19 pandemic: Global predictive modelling to inform surgical recovery plans. Br J Surg 2020;107(11):1440-1449. https://do org $/ 10.1002 /$ bjs. 11746
8. South African Society of Anaesthesiologists. Recommendations for the management of anaesthesia and surgery for elective procedures. SASA newsletter, 25 March 2020. https://www.samedical.org/files/ and surgery for elective procedures. SASA newsletter, 25 March 2020. https://wWw.samedical.org/files/
covid19/doctor_resource/SASA\%20ElectiveSurgerySASACOVID19_25.03.2020_637207485661555627. dff (accessed 18 June 2020).

9. Pranchand VN, Milner R, Angelos P, et al. Medically necessary, time-sensitive procedures: Scoring system to ethically and efficiently manage resource scarcity and provider risk during the COVID-19 pandemic. J Am Coll Surg 2020;231(2):281-288. https://doi.org/10.1016/j.jamcollsurg.2020.04.01

10. Ing E, Xu Q, Salimi A, Toroun N. Physician deaths from corona virus (COVID-19) disease. Occup Med 2020;70(5):370-374. https://doi.org/10.1093/occmed/kqaa08

11. Rubino S, Kelvin N, Bermejo-Martin J, Kelvin D. As COVID-19 cases, deaths and fatality rates surge in Italy, underlying causes require investigation. J Infect Dev Ctries 2020;14(3):265-267. https://doi, org $/ 10.3855 /$ jidc. 12734

Accepted 13 November 2020 